\title{
Tenderness Over the Hyoid Bone Can Indicate Epiglottitis in Adults
}

\author{
Hiroshi Ehara, MD
}

\begin{abstract}
Adult acute epiglottitis is a rare but life-threatening disease caused by obstruction of the airway. The symptoms and signs of this disease may be nonspecific without apparent airway compromise. We encountered 3 consecutive cases of adult patients with this disease in a single 5-month period in one physician's office. In all cases, physical examination revealed tenderness of the anterior neck over the hyoid bone. These observations assisted us in identifying this rare disease quickly. We suggest that tenderness over the hyoid bone should raise suspicion of adult acute epiglottitis. (J Am Board Fam Med 2006;19: 517-20.)
\end{abstract}

Adult acute epiglottitis is an inflammatory disease of the epiglottis and adjacent structures resulting from infection. It can be a rapidly fatal condition because of the potential for sudden upper airway obstruction. Early recognition of acute epiglottitis is therefore of the utmost importance in minimizing morbidity and mortality.

Unfortunately, misdiagnosis occurs in $23 \%$ to $31 \%$ of the cases of adult acute epiglottitis. ${ }^{1-3}$ During a single 5-month period, we encountered 3 cases of the disease in our physician's office. Physical examination revealed tenderness of the middle anterior neck, especially over the hyoid bone, in all patients. This sign enabled us to diagnose this uncommon disease quickly. All the patients steadily improved without the need for interventional airway support. Here we propose that tenderness over the hyoid bone should be considered a major sign in the diagnosis of adult acute epiglottitis.

\section{Case Reports \\ case 1}

A 55-year-old woman presented to the Ehara Clinic in July 2004 complaining of a severe sore throat that had begun the previous day, and with a fever of $37.5^{\circ} \mathrm{C}$. She complained of dysphagia and odynophagia. She experienced a loss of vocal

This article was externally peer-reviewed.

Submitted 9 January 2006; revised 6 April 2006; accepted 7 April 2006.

From Ehara Clinic, Fuchu-shi, Tokyo, Japan.

Conflict of interest: none declared.

Corresponding author: Hiroshi Ehara, MD, Ehara Clinic, 3357 Wakamatsu cho, Fuchu-shi, Tokyo, 1830005 Japan (E-mail: eharaiin@clock.ocn.ne.jp). power, and talking aggravated her sore throat. At admission, the patient did not seem to be critically ill. Her voice was neither muffled nor hoarse. The vital signs indicating the nature of her condition were as follows: body temperature, $37.0^{\circ} \mathrm{C}$ (axillary); blood pressure, 90/64 mm Hg; pulse, 64/min; respirations, 24/min; peripheral oxygen saturation, $97 \%$. At this point, these findings were not sufficient to indicate any serious diseases. The pharynx was slightly erythematous. Dyspnea, stridor, and drooling were not found. The bilateral upper cervical lymph nodes were mildly swollen. Careful neck palpation revealed tenderness of the anterior median neck. The lungs were clear. The results of the remainder of the physical examination were within normal limits. A lateral neck radiograph revealed an enlargement of the epiglottis and a narrowing of the vallecula.

The patient was referred to the hospital to be seen by an otolaryngologist. Fiberoptic laryngoscopy revealed that the lingual surface of the epiglottis was swollen, but the laryngeal surface and the arytenoids remained unswollen. The patient was given hydrocortisone sodium succinate intravenously (300 mg/day) and instructed to take $60 \mathrm{mg}$ of loxoprofen sodium 3 times a day. Here, it was assumed that the patient was very unlikely to develop airway obstruction, so she was carefully followed as an outpatient. One day after admission, her severe pain had resolved. Laryngoscopy revealed a slightly swollen epiglottis. Hydrocortisone administration was tapered to $200 \mathrm{mg} /$ day, and the patient was instructed to take $100 \mathrm{mg}$ of cefditoren pivoxil orally 3 times a day, and to complete a 7 -day course of antibiotics. 


\section{Case 2}

A 32-year-old man presented to the emergency department of a hospital one night in October 2004 complaining of a sore throat that had begun that evening, associated with a fever of $37.6^{\circ} \mathrm{C}$. The patient was discharged with a diagnosis of pharyngitis and instructed to take $270 \mathrm{mg}$ of salicylamide, $150 \mathrm{mg}$ of acetaminophen, $60 \mathrm{mg}$ of anhydrous caffeine, $13.5 \mathrm{mg}$ of promethadine methylene disalicylate, and $100 \mathrm{mg}$ of ibuprofen 3 times a day. The next morning, the patient presented to the Ehara Clinic complaining that his sore throat had gradually worsened over the previous 16 hours. Odynophagia seemed to have progressed and was accompanied by a fever of $38.5^{\circ} \mathrm{C}$. At admission, the patient presented with a cold compress applied to his mid upper anterior neck. He complained of significant pain at that site. During physical examination, the patient showed no sign of anxiety, and there was no evidence of a muffled voice, dyspnea, stridor, or drooling. His vital signs were: blood pressure, 108/70 mm Hg; pulse, 114/min; respirations, $22 / \mathrm{min}$; temperature, $38.1^{\circ} \mathrm{C}$ (axillary). His pharynx was slightly reddened. He experienced a tenderness of the middle anterior neck, especially over the hyoid bone, on which a cold compress was centrally applied. Cervical lymph nodes were not palpable. A chest examination proved unremarkable. Findings on a lateral neck radiograph included the "thumb sign" (swelling of the epiglottis) and the "vallecula sign" (a decrease in vallecular air space) (Figure 1).

The patient was subsequently transferred to the hospital. Flexible laryngoscopy revealed that the lingual surface of the epiglottis was swollen, but the laryngeal surface and the arytenoids remained unswollen. The patient was given the following medication intravenously: $2 \mathrm{~g} /$ day piperacillin, 1.2 $\mathrm{g} /$ day clindamycin, and $500 \mathrm{mg}$ /day hydrocortisone sodium succinate. The following day, the hydrocortisone was tapered to $300 \mathrm{mg} /$ day. Two days after admission, laryngoscopy disclosed a marked decrease in swelling of the epiglottis and the patient was discharged. Four days after the discharge, his symptoms had completely disappeared. Laryngoscopy revealed a normal epiglottis.

\section{Case 3}

In December 2004, a 29-year-old man presented with a severe sore throat that had begun the day before, and a chill. Odynophagia had developed

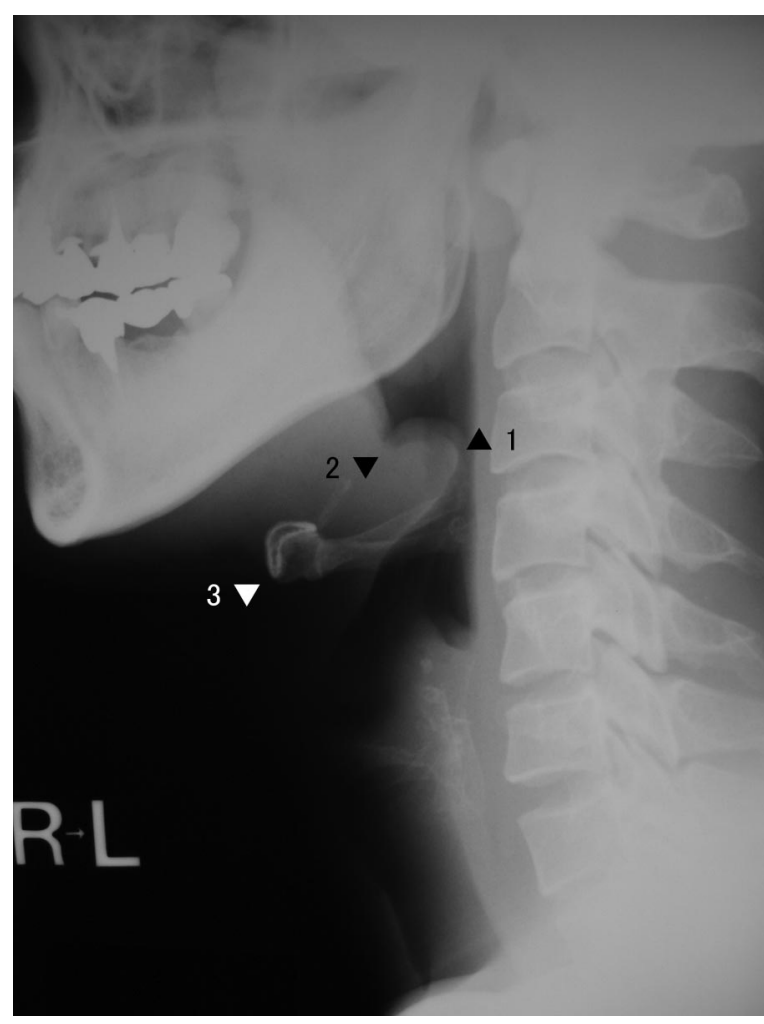

Figure 1. Lateral soft tissue radiograph of the neck in case 2: thumb sign: rounded configuration of the epiglottis (1); vallecula sign: narrowing of the vallecula (2); hyoid bone (3).

and worsened, and he experienced difficulty swallowing saliva. During physical examination, he displayed no sign of anxiety. We found no dyspnea, stridor, hoarseness, or drooling. His temperature was $37.1^{\circ} \mathrm{C}$ (axillary). The pharynx was slightly injected, and the cervical lymph nodes were not palpable. He complained of severe pain in the upper median anterior neck. His upper anterior neck was tender to palpation, especially over the hyoid bone. His chest was clear. A lateral cervical radiograph disclosed both thumb and vallecula signs.

The patient was subsequently admitted to the hospital. Flexible fiberoptic laryngoscopy disclosed that the lingual surface of the epiglottis was reddened and swollen, but the laryngeal surface and the arytenoids remained unswollen. Laboratory tests revealed a white blood cell count of $14.5 \times 10^{3}$ $\mu \mathrm{L}$ cells and a C-reactive protein level of $0.3 \mathrm{mg} /$ $\mathrm{dL}$. The patient was intravenously administered 2 $\mathrm{g} /$ day flomoxef. Four days after admission, laryngoscopy disclosed a normal epiglottis, and the patient was discharged. 


\section{Discussion}

Studies that have focused on adult acute epiglottitis reveal that $88 \%$ to $100 \%$ of patients presented with a sore throat, ${ }^{1-9}$ dysphagia was observed in $75 \%$ to $92 \%, 2,5,6-8,10$ and odynophagia was evident in $68 \%$ to $100 \%$ of the patients. ${ }^{1-4,8,10,11}$ Although only $20 \%$ to $44 \%$ of patients presented with dyspnea, ${ }^{1-3,5,8,10,11}$ hoarseness or muffled voice were found in $13 \%$ to $79 \%$, drooling in $7 \%$ to $41 \%$, and stridor in a further $7 \%$ to $27 \% .^{1-9,11}$ Thus, most adults who have epiglottitis complain only of a sore throat and odynophagia/dysphagia without displaying any signs or symptoms of airway obstruction. ${ }^{1-5,8-11}$ Dyspnea, stridor, drooling, and muffled voice were present when interventional airway support was required. Dyspnea, stridor, and drooling have been recognized as reliable warning signs of impending airway collapse during an episode of acute epiglottitis. ${ }^{1,3-5,10}$

The severity of sore throat in most patients may be greater than the oropharyngeal observations suggest. In $80 \%$ of cases, the pharynx may seem normal or slightly reddened without severe pharyngitis. ${ }^{6}$ The clinician should suspect epiglottitis in patients with severe sore throat and odynophagia, even in circumstances where oropharyngeal exam-

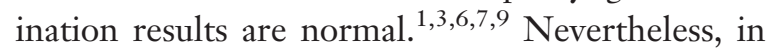
routine practice, it may be difficult for physicians to make a correct diagnosis of this rare disease, based only on subjective statements in the absence of any positive objective physical evidence.

The 3 patients highlighted in this study presented with severe sore throats and odynophagia but displayed no signs or symptoms of airway compromise. These patients showed only slight erythema of the pharynx, which was disproportionate to their symptoms. Given only this physical evidence, it is possible to miss the diagnosis. Careful palpation identified tenderness of the upper middle anterior neck. This was the key positive objective finding indicating the epiglottitis presenting with "sore throat." This was especially evident in case 2, in which the patient had applied a cold compress to his upper mid anterior neck centrally over the hyoid bone. Given these circumstances, it was relatively easy to have a high degree of suspicion of acute epiglottitis. Based on our experience with this case, by performing careful manipulation, we were confidently able to predict acute epiglottitis from the tenderness over the hyoid bone in the patient described in case 3 .

Although many studies have been conducted by specialists who can easily perform direct visualization of the larynx using a laryngoscope, it is uncertain how much attention has been paid to anterior neck tenderness. As only a few literature references have documented this sign, we suspect that tenderness over the hyoid bone has not been sufficiently evaluated as a major sign for the presence of epiglottitis.

In a retrospective study of case series, Frantz et al found that 41 of 129 patients (32\%) had cervical tenderness and adenopathy. ${ }^{3}$ In a similar study, Mayo-Smith et al reported that 79 of 100 adult cases $(79 \%)$ had tenderness of the anterior neck. ${ }^{5}$ Shapiro et al reported prospectively that 7 of 8 patients $(87 \%)$ had significant cervical tenderness and swelling, ${ }^{12}$ and Khilanani et al, Cohen, and Mace reported that 3 of 4,2 of 3 , and 5 of 5 patients, respectively, exhibited tenderness of the anterior neck. ${ }^{6,13,14}$ Khilanani et al and Cohen stressed that anterior neck tenderness should lead to a suspicion of adult acute epiglottitis. ${ }^{6,13}$ Cohen, in particular, remarked that tenderness to palpation of the neck over the hyoid complex may be the clue to diagnosis of this disease. ${ }^{13}$

Adult acute epiglottitis is often referred to as supraglottitis because inflammation is generally not confined to the epiglottis, but can also affect other structures. In addition to the epiglottis, supraglottic structures such as the base of tongue and the vallecula are also frequently affected. ${ }^{12}$ Anatomically, the hyoid bone is bound to the epiglottis by the hyoepiglottic ligament. Acute inflammation of the epiglottis and supraglottis occurs just behind the hyoid bone (Figure 2). The occurrence of phenomena such as uvular and tonsillar edema on an oropharyngeal examination, does not rule out a concomitant supraglottitis or epiglottitis. ${ }^{12}$ Although the absence of tenderness over the hyoid bone does not exclude epiglottitis, the finding may increase the suspicion of this rare disease, regardless of the oropharyngeal observations.

It is a matter of course that the gold standard for identification of epiglottitis is by flexible endoscopy, the use of which, if possible, is encouraged among general practitioners. ${ }^{8,11}$ Nevertheless, in patients in whom a laryngoscopy cannot be performed and whose respiratory status is secure, careful manipulation, not to upset the patient, and lat- 


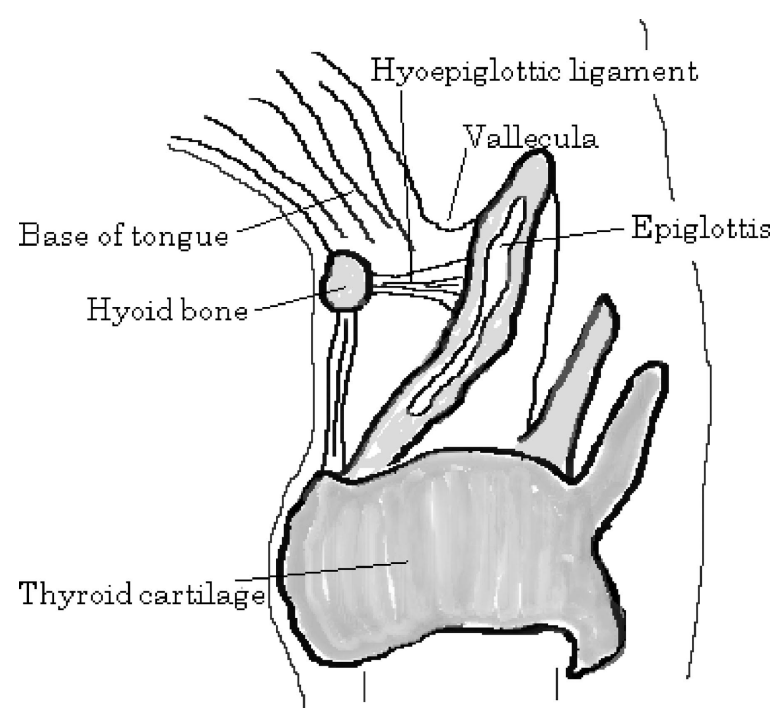

Figure 2. Anatomic association between the epiglottis and the hyoid bone; the epiglottis, valeculla, and base of the tongue are just behind the hyoid bone.

eral neck soft-tissue radiographs can be expected to provide helpful information as our series $\mathrm{did} .{ }^{12,15}$ When the physician remains suspicious of epiglottitis on clinical grounds but diagnostic findings are not detected on radiographs, immediate referral to an otolaryngologist for further evaluation with laryngoscopy is necessary. ${ }^{3,5}$ If the patient is already in respiratory distress, the patient must be transferred to a hospital where appropriate airway management should be performed before the diagnosis can be made by inspection to prevent the patient from inducing an inspiratory crisis.

\section{Conclusion}

We emphasize the importance of tenderness over the hyoid bone as a guide to suspicion of adult acute epiglottitis in patients who complain of severe sore throat and odynophagia/dysphagia. For general practitioners in routine practice, prospective evaluation of tenderness over the hyoid bone will be necessary to determine its effectiveness as a major sign of adult acute epiglottitis.

The author thanks Dr. Kazuya Abe (Tokyo Metropolitan Fuchu Hospital) for both presenting case records and suggesting his management of epiglottitis; Dr. Kouichi Yamauchi (Dept. of Otolaryngology, Head and Neck Surgery, Kyorin University) for presenting a case record; and Dr. Yutaka Imauchi (Dept. of Otolaryngology, Japanese Red Cross Musashino Hospital) for presenting a case record.

\section{References}

1. Berger G, Landau T, Berger S, Finkelstein Y, Bernheim J, Ophir D. The rising incidence of adult acute epiglottitis and epiglottic abscess. Am J Otolaryngol $2003 ; 24: 374-83$.

2. Mustoe T, Strome M. Adult epiglottitis. Am J Otolaryngol 1983;4:393-9.

3. Frantz TD, Rasgon BM, Quesenberry CP Jr. Acute epiglottitis in adults: analysis of 129 cases. JAMA 1994;272:1358-60.

4. Chang YL, Lo SH, Wang PC, Shu YH. Adult acute epiglottitis: experiences in a Taiwanese setting. Otolaryngol Head Neck Surg 2005;132:689-93.

5. Mayo-Smith MF, Spinale JW, Donskey CJ, Yukawa M, Li RH, Schiffman FJ. Acute epiglottitis: an 18year experience in Rhode Island. Chest 1995;108: $1640-7$.

6. Khilanani U, Khatib R. Acute epiglottitis in adults. Am J Med Sci 1984;287:65-70.

7. Wong EY, Berkowitz RG. Acute epiglottitis in adults: the Royal Melbourne Hospital experience. ANZ J Surg 2001;71:740-3.

8. Solomon P, Weisbrod M, Irish JC, Gullane PJ. Adult epiglottitis: the Toronto Hospital experience. J Otolaryngol 1998;27:332-6.

9. Stanley RE, Liang TS. Acute epiglottitis in adults (the Singapore experience). J Laryngol Otol 1988; 102:1017-21.

10. Hebert PC, Ducic Y, Boisvert D, Lamothe A. Adult epiglottitis in a Canadian setting. Laryngoscope 1998;108:64-9.

11. Torkkeli T, Ruoppi P, Nuutinen J, Kari A. Changed clinical course and current treatment of acute epiglottitis in adults: a 12-year experience. Laryngoscope 1994;104:1503-6.

12. Shapiro J, Eavey RD, Baker AS. Adult supraglottitis: a prospective analysis. JAMA 1988;259:563-7.

13. Cohen EL. Epiglottitis in adults. Ann Emerg Med 1984;13:620-3.

14. Mace SE. Acute epiglottitis in adults. Am J Emerg Med 1985;3:543-50.

15. Ducic Y, Hebert PC, MacLachlan L, Neufeld K, Lamothe A. Description and evaluation of the vallecula sign: a new radiologic sign in the diagnosis of adult epiglottitis. Ann Emerg Med 1997;30:1-6. 